lustitia Socialis. Revista Arbitrada de Ciencias Jurídicas.

Año V. Vol. V. №3. Edición Especial. 2020-III:

Universidad Regional Autónoma de los Andes

Hecho el depósito de Ley: FA2016000064

ISSN: 2542-3371

FUNDACIÓN KOINONIA (F.K). Santa Ana de Coro, Venezuela

Marco Fernando Saltos-Salgado; Carlos Alfonso Grijalva-Terán

http://dx.doi.org/10.35381/racii.v5i3.1131

\title{
Derechos de los grupos de atención prioritaria en el Instituto Ecuatoriano de Seguridad Social
}

Rights of priority care groups in the Ecuadorian Social Security Institute

\author{
Marco Fernando Saltos-Salgado \\ us.marcosaltos@uniandes.edu.ec \\ Universidad Regional Autónoma de los Andes, Santo Domingo \\ Ecuador \\ https://orcid.org/0000-0003-1679-9243 \\ Carlos Alfonso Grijalva-Terán \\ us.carlosgrijalva@uniandes.edu.ec \\ Universidad Regional Autónoma de los Andes, Santo Domingo \\ Ecuador
}

Recibido: 31 de octubre de 2020

Revisado: 10 de octubre de 2020

Aprobado: 05 de diciembre de 2020

Publicado: 10 de diciembre de 2020 


\title{
RESUMEN
}

La siguiente investigación se basó en la observación a la vulneración de los derechos de los grupos de atención prioritaria en el Hospital del Instituto Ecuatoriano de Seguridad Social de la ciudad de Santo Domingo. Desde la perspectiva cuantitativa y a través de una investigación de campo se logró obtener y recolectar datos mediante un cuestionario aplicado a los usuarios del servicio, los adultos mayores y experiencia a través de sus familiares, para lo cual se elaboró diversas preguntas que arrojan respuestas precisas, con el único fin de obtener información necesaria y efectiva para la investigación, con adultos mayores en el Hospital antes mencionado. También se utilizó la investigación bibliográfica-documental, con el fin de obtener mayor información. En conclusión, se establece la necesidad de entablar los canales de atención prioritaria y canal de capital humano, cuyo resultado es la existencia de personal capacitado en atención de enfermedades degenerativas y catastróficas en el ámbito geriátrico.

Descriptores: Envejecimiento; derecho a la salud; derechos humanos. (Palabras tomadas del Tesauro UNESCO).

\begin{abstract}
The following investigation was based on the observation of the violation of the rights of priority care groups in the Hospital of the Ecuadorian Institute of Social Security in the city of Santo Domingo. From the quantitative perspective and through a field investigation, data was obtained and collected through a questionnaire applied to the users of the service, the elderly and experience through their relatives, for which various questions were elaborated that provide precise answers, with the sole purpose of obtaining necessary and effective information for the investigation, with older adults in the aforementioned Hospital. Bibliographic-documentary research was also used in order to obtain more information. In conclusion, the need to establish priority care channels and human capital channel is established, the result of which is the existence of personnel trained in care of degenerative and catastrophic diseases in the geriatric field.
\end{abstract}

Descriptors: Aging; right to health; human rights. (Words taken from the UNESCO Thesaurus). 
lustitia Socialis. Revista Arbitrada de Ciencias Jurídicas.

Año V. Vol. V. №3. Edición Especial. 2020-III:

Universidad Regional Autónoma de los Andes

Hecho el depósito de Ley: FA2016000064

ISSN: 2542-3371

FUNDACIÓN KOINONIA (F.K). Santa Ana de Coro, Venezuela

Marco Fernando Saltos-Salgado; Carlos Alfonso Grijalva-Terán

\section{INTRODUCCIÓN}

El Instituto Ecuatoriano de Seguridad Social (IESS) es una entidad autónoma que se rige por los principios de solidaridad, obligatoriedad, universalidad, equidad, eficiencia, subsidiaridad, suficiencia, transparencia y participación, y con un derecho irrenunciable de todas las personas tal como lo menciona la Constitución de la República del Ecuador, que tiene por objetivo principal la atención médica a grupos de interés que por su situación de salud requieren de un tratamiento y trato especial.

El Estado ecuatoriano tiene el deber fundamental a través de las políticas públicas garantizar los derechos de las personas adultas mayores; promoviendo la creación de centros especializados y hospitales geriátricos que brinden una esmerada atención, si bien es cierto que el crecimiento demográfico denota que cada vez se acrecienta la población de los adultos mayores y por ende cobra mayor importancia para las autoridades competentes, en que se los considere como lo determina la Constitución como el grupo de atención prioritaria.

La población mundial está envejeciendo rápidamente. Entre 2015 y 2050 la proporción de la población mundial mayor de 60 años se multiplicará casi por dos, pasando del $12 \%$ al 22\%, la inmensa mayoría de los cuales vivirán en países de ingresos medianos o bajos. En el Ecuador existen: 1.049.824 personas mayores de 65 años que representa el 6,5\% de la población total. En el año 2020 será del 7,4\%. Para el año 2054 se prevé que representen el $18 \%$ de la población. Para las mujeres la esperanza de vida será mayor con 83,5 años comparado con los 77,6 años de los hombres. Por otro lado, se enfatiza que la violencia hacia el adulto mayor no puede limitarse solo a los aspectos físico, emocional, sexual o patrimonial también existe la violencia financiera, por negligencia, negación a brindarle ayuda y otras formas más, lo cual sería materia de análisis. (Chóez-Chiliguinga \& otros, 2019)

La Ley del Anciano expedida en el año 1991, tiene como objetivo primordial garantizar el derecho a un nivel de vida que aseguren la salud corporal, psicológica, alimentación, vestido, vivienda, asistencia médica, la atención geriátrica y gerontológica integral para una existencia útil y decorosa. (Ley del Anciano, 1991) 
Con estos antecedentes, si bien es cierto que se han logrado importantes avances constitucionales en materia de reconocimiento y garantía de los derechos de este grupo de atención prioritaria, no existe en la actualidad una adecuada legislación que permita atender y proteger, en forma prioritaria e integral, a las personas adultas mayores en nuestro país. El Ministerio de Salud Pública de Ecuador, en su calidad de autoridad sanitaria nacional, está implementando cambios estructurales en el sector salud, que se enmarcan en el proceso de la revolución ciudadana que el gobierno nacional viene impulsando a partir de 2007. Desde esta perspectiva, la salud es reconocida como un derecho fundamental garantizado por el Estado, y se constituye en un eje estratégico para el desarrollo y el logro del buen vivir. (Batista-Pereda \& Otros, 2016, p.532)

Debemos de considerar que en la actualidad los adultos mayores han sido excluido de la sociedad, y al referirse a los servicios de salud especialmente al tratarse de enfermedades catastróficas el Estado no ha priorizado esta atención, puesto que en muchos centros de salud no existe el tratamiento y el personal adecuado para comprender su necesidad, así como también a la sobrepoblación que atienden los profesionales de la salud y el no contar con los recursos necesarios para poder hacerlo efectivo y eficaz los que les obliga a muchos a estar en sus hogares utilizando medios alternativos para aliviarse sin que reciban la atención medica requerida.

El Estado es el obligado a velar por la salud de este grupo poblacional, es uno de sus derechos marcado en la Constitución de la República del Ecuador, por lo que deberá emplear mecanismos para que se priorice la salud de los adultos mayores, a través de las políticas públicas como se menciona en el Art. 359 de dicho cuerpo legal. Lastimosamente en nuestro país el $75.34 \%$ de la población mayor a 65 años no cuenta con afiliación a un seguro de salud; y solo el $24.66 \%$ de la población está afiliada al IESS, estas cifras nos permiten analizar la debilidad de la seguridad social, y la falta de recursos para la atención de todos los adultos mayores que acuden por un tratamiento médico.

Es importante considerar que en nuestra Constitución existe un derecho como es el de Participación Ciudadana, en donde todos los ciudadanos debemos acudir para que 
se consideren las propuestas y proyectos de ley que se presenten, los que deberán ser analizados por experto en la materia y modificarlos o reformarlos con el afán de que se escuche la voz del poder público (pueblo), tal como se hace mención en el Art. 137 inciso segundo Es así que debemos de ser consciente de que si no se está cumplimiento lo establecido en la Constitución se debe hacer conocer, en el caso del Instituto Ecuatoriano de Seguridad Social de la ciudad de Santo Domingo no ha acotado la propuesta del hospital Teodoro Maldonado Carbo de especificar un horario para la atención personalizada de los adultos mayores, es menester de las autoridades realizar un control para su cumplimiento y evitar con ellos el caos y la inseguridad de quienes necesitan atención.

El Instituto Ecuatoriano de Seguridad Social (IESS) es una entidad autónoma que se rige por los principios de solidaridad, obligatoriedad, universalidad, equidad, eficiencia, subsidiaridad, suficiencia, transparencia y participación, y con un derecho irrenunciable de todas las personas tal como lo menciona la Constitución de la República del Ecuador, que tiene por objetivo principal la atención médica a grupos de interés que por su situación de salud requieren de un tratamiento y trato especial.

En cuanto a la atención prioritaria, haciendo referencia a los adultos mayores que padecen de enfermedades catastróficas como hipertensión pulmonar solicitan citas por ampliación de agenda y no necesitan llamar al Call Center para que se les asigne una, viendo esta necesidad se acordó en reunión dictar una resolución desde el Hospital Teodoro Maldonado Carbo, en que se realice un servicio personalizado con disposición de horario los días viernes de 12:00 a 14:00 para atención exclusiva de estas personas.

La situación problémica radica en que en la ciudad de Santo Domingo no se ha integrado el último acuerdo dictado, lo que ha dado paso a que exista la vulneración del derecho a la salud, ya que lo paciente tienen que trasladarse a ciudades donde cuentan con esta atención, dentro del día y horario establecido.

En el artículo 359 de la Constitución de la República del Ecuador, “...garantizará la promoción, prevención, recuperación y rehabilitación con lo que respecta al derecho a la salud..." y en la Ley del Anciano, en el capítulo III "Art.7.- Los servicios médicos 
de los establecimientos públicos y privados, contarán con atención geriátrico - gerontológico para la prevención, el diagnóstico y tratamiento de las diferentes patologías de los ancianos, y su funcionamiento se regirá por lo dispuesto en la presente Ley, su Reglamento y el Código de la Salud", con lo que se demuestra claramente que tanto la Constitución como la Ley del Anciano vinculan a las instituciones de salud tanto públicas como privadas para su atención y tratamiento lo que hace el Instituto Ecuatoriano de Seguridad Social vulnerar este derecho y garantía que el Estado brinda al grupo prioritario.

Al realizar un análisis actualizado del porque se considera una mala atención médica y vulneración del derecho a la salud al grupo prioritario como son los adultos mayores se puede considerar que es debido a la mala administración por no acotar y cumplir con disposiciones que ellos consideran no de mucha importancia dentro de los dispensarios $u$ hospital en los dirigen, $y$ hacen hincapié a la falta de recursos económicos con los que no cuenta para buscar una disculpa de su no cumplimiento. Si se hablara del Buen Vivir, esto lo definiríamos como un principio constitucional basado en el Sumak Kawsay, que recoge una visión centrada en el ser humano, otorgándole de ser la satisfacción de las necesidades en consecución de una calidad de vida, dando con ello una libertad potencializada en reales oportunidades de los individuos donde se amplían y florecen de manera simultánea la paz y armonía con la naturaleza, es sin duda algo que hace conocer que los derechos son respetados por estar plasmado en la Constitución, pero la realidad es diferente, puesto que al tratarse del grupo prioritario (adultos mayores), no se cumple con todo lo que hace referencia dentro de sus derechos y garantías.

Hay que considerar que este grupo de atención prioritaria, ha realizado un aporte muy considerable para ser dignos de una buena atención, de un horario especifico y acorde a sus necesidades para ser atendidos, sin que se les permita una cita agendada que nunca llega, las autoridades competentes deben de ser quienes estén frente a que se cumpla con todo lo que dispone la Constitución, y evitar que estas personas que desean una atención médica especializada viajen a lugares donde no tiene un familiar para que les reciba hasta cumplir con sus tratamientos. 
El objetivo general de la presente investigación fue determinar la necesidad de incrementar la atención del adulto mayor en los centros de cuidado del IESS.

\section{METODOLOGÍA}

Desde la perspectiva cuantitativa y a través de una investigación de campo se logró obtener y recolectar datos directamente de la realidad en cuanto a la atención de los adultos mayores en el Hospital del Instituto de Seguridad Social de la ciudad de Santo Domingo. También se utilizó la investigación bibliográfica-documental, con el fin de obtener mayor información mediante documentos, libros, datos de investigaciones anteriormente realizadas, lo que permitió conseguir la información necesaria para fundamentar la investigación. El método analítico-sintético facilitó el análisis minucioso del problema de estudio como a la vez obtener una síntesis que permitió profundizar la respectiva problemática que existe, con lo cual se logró determinar las consecuencias sociales que ocasiona la no aplicación de un horario específico para la atención de los adultos mayores. Como técnica e instrumento de la investigación se utilizó la encuesta las cuales constituyo un instrumento breve y muy útil para obtener información de la muestra designada.

Por consiguiente la población constituyo en este caso la muestra; lo cuales fueron encuestados veinte usuarios aplicando para la selección de esta muestra aleatoria el muestreo no probabilístico por conveniencia en consideración de que estos usuarios tienen un amplio conocimiento acerca de la falta de organización para la atención de los adultos mayores y experiencia a través de sus familiares, para lo cual se elaboró diversas preguntas que arrojan respuestas precisas, con el único fin de obtener información necesaria y efectiva para la investigación. 
lustitia Socialis. Revista Arbitrada de Ciencias Jurídicas.

Año V. Vol. V. №3. Edición Especial. 2020-III:

Universidad Regional Autónoma de los Andes

Hecho el depósito de Ley: FA2016000064

$$
\text { ISSN: 2542-3371 }
$$

FUNDACIÓN KOINONIA (F.K). Santa Ana de Coro, Venezuela

Marco Fernando Saltos-Salgado; Carlos Alfonso Grijalva-Terán

\section{RESULTADOS}

A continuación, se exhibe las tablas de los resultados obtenidos:

Tabla 1

Encuestas usuario del IESS del cantón Santo Domingo.

\begin{tabular}{|c|c|c|}
\hline PREGUNTAS & ANÁLISIS & PORCENTAJE \\
\hline $\begin{array}{l}\text { ¿Usted como usuario cree } \\
\text { que tiene una buena } \\
\text { atención médica? }\end{array}$ & $\begin{array}{c}\text { Los encuestados manifestaron } \\
\text { que ellos no todos son } \\
\text { atendidos } \\
\text { y que si van por emergencia } \\
\text { tienen que ser de gr avedad }\end{array}$ & $20 \%$ \\
\hline $\begin{array}{l}\text { ¿Cuándo un adulto mayor de su } \\
\text { familia necesita ayuda médica es } \\
\text { atendido urgentemente en esta } \\
\text { casa de salud? }\end{array}$ & $\begin{array}{l}\text { Si el caso es de gravedad sí, } \\
\text { pero si puede esperar a que los } \\
\text { médicos se desocupen tienen que } \\
\text { esperar. }\end{array}$ & $20 \%$ \\
\hline $\begin{array}{l}\text { ¿Cree usted que debe exist ir un } \\
\text { horario para la atención de los } \\
\text { adultos mayores solo para ellos? }\end{array}$ & $\begin{array}{c}\text { Si sería lo mejor, así todas las } \\
\text { semanas ellos tendrian su } \\
\text { control } \\
\text { como se lo merecen }\end{array}$ & $20 \%$ \\
\hline $\begin{array}{c}\text { ¿Cree usted que el personal } \\
\text { médico es el adecuado para la } \\
\text { atención de los adultos } \\
\text { mayores? }\end{array}$ & $\begin{array}{l}\text { No porque muchos de } \\
\text { ellos piensan que si los } \\
\text { adultos } \\
\text { mayores acuden a la consulta es } \\
\text { por decir que tienen medicina }\end{array}$ & $20 \%$ \\
\hline $\begin{array}{l}\text { ¿Considera usted que los } \\
\text { adultos mayores no deban } \\
\text { viajar a otra ciudad para } \\
\text { continuar su tratamiento? }\end{array}$ & $\begin{array}{l}\text { Claro que no ellos no tienen } \\
\text { la edad ya para andar de un } \\
\text { lugar } \\
\text { para otro, existiendo este centro } \\
\text { de salud que puede brindarle }\end{array}$ & $20 \%$ \\
\hline \multicolumn{2}{|c|}{ TOTAL } & $100 \%$ \\
\hline
\end{tabular}

Fuente: Usuarios del IESS del cantón Santo Domingo

Elaborado: Carlos Alfonso Grijalva Terán(2021) 
lustitia Socialis. Revista Arbitrada de Ciencias Jurídicas.

Año V. Vol. V. №3. Edición Especial. 2020-III:

Universidad Regional Autónoma de los Andes

Hecho el depósito de Ley: FA2016000064

ISSN: 2542-3371

FUNDACIÓN KOINONIA (F.K). Santa Ana de Coro, Venezuela

Marco Fernando Saltos-Salgado; Carlos Alfonso Grijalva-Terán

\section{DISCUSIÓN}

Los resultados de las encuestas han demostrado que los usuarios necesitan de urgencia que se les otorgue un día y hora especial para la atención de los adultos mayores, ya que esto ha generado que muchos de ellos emigren a otras ciudades pese a tener dificultades para movilizarse y que por no encontrar la solución dentro del lugar de su residencia se ven obligado a buscar por otro lado sus tratamiento y atención médica.

La Constitución de la República del Ecuador, así como la Ley del Anciano definen la atención médica a los adultos mayores como prioritaria, y que ante cualquier dolencia o urgencia de este grupo debe ser atendida de inmediato, pero en la actualidad este grupo tiene que esperar meses para ser atendido, y acudir a otro lugar para recibir su tratamiento y calmar sus dolencias.

De la investigación desarrollada también se dio a conocer como resultado que existe un déficit de ingreso para compensar la atención médica dentro de los hospitales del Instituto Ecuatoriano de Seguridad Social, lo que ha conllevado a que muchos profesionales renuncien a sus puestos o sean removidos de los mismos por falta de fondo, este también es un problema gigantesco que perjudica de sobre manera la atención médica en estos centros de salud, este desequilibrio institucional se merma aún más con la restricción de turnos a sus usuarios, siendo así considerado la vulneración a un derecho importante como el de la salud.

Al existir la vulneración de este derecho que garantiza el Estado y conforme se ha visto reflejado en los resultados de la investigación desarrollada, constituyendo precisamente este derecho fundamental constitucional uno de los pilares más importantes que el Estado garantiza a los ciudadanos y ciudadanas en especial al grupo de atención prioritaria, se encuentra legalmente reconocido en la Declaración Universal de Derechos Humanos que dice en su preámbulo: Reconociendo que la persona, a medida que envejece, debe seguir disfrutando de una vida plena, independiente y autónoma, con salud, seguridad, integración y participación activa en las esferas económica, social, cultural y política de sus sociedades; .. Decididos a incorporar y dar prioridad al tema del envejecimiento en las políticas públicas, así como 
a destinar y gestionar los recursos humanos, materiales y financieros para lograr una adecuada implementación y evaluación de las medidas especiales puestas en práctica... (OEA, 2012).

Los resultados demuestran efectivamente que por la falta de atención medica específica para los adultos mayores, éstos tienen que acudir a otras ciudades a recibir su tratamiento o realizarse su control médico necesario, por lo que afecta considerablemente su salud, ocasionando una vulneración al derecho de la misma por parte del hospital del Instituto Ecuatoriano de Seguridad Social, lo que se ve reflejado en las estadísticas de atención realizadas.

Lo que se motivó para realizar el presente artículo científico es la vulneración a ese derecho primordial, principal y fundamental de los adultos mayores como es el de la salud, pese a ser considerado el grupo prioritario dentro de la Constitución y Convenios Internacionales tal como se lo describe de la siguiente manera: Art. 36.Las personas adultas mayores recibirán atención prioritaria y especializada en los ámbitos público y privado, en especial en los campos de inclusión social y económica, y protección contra la violencia. Se considerarán personas adultas mayores aquellas personas que hayan cumplido los sesenta y cinco años de edad, Art. 37.- El Estado garantizará a las personas adultas mayores los siguientes derechos: 1. La atención gratuita y especializada de salud, así como el acceso gratuito a medicinas... (Constitución, 2018)

En el Artículo 19.- Derecho a la salud: La persona mayor tiene derecho a su salud física y mental, sin ningún tipo de discriminación.

Los Estados Parte deberán diseñar e implementar políticas públicas intersectoriales de salud orientadas a una atención integral que incluya la promoción de la salud, la prevención y la atención de la enfermedad en todas las etapas, y la rehabilitación y los cuidados paliativos de la persona mayor a fin de propiciar el disfrute del más alto nivel de bienestar, físico, mental y social. (OEA, 2012)

Lo que conlleva analizar que la atención de la salud de los adultos mayores se encuentra garantizada tanta en nuestra Constitución como en la Convención de los Derechos Humanos, lo que hace que se ve vulnerado en los centros de salud ya 
sea por la mala administración, por falta de recursos económicos o simplemente por falta de interés de los profesionales a cargo de estos centros. Resulta necesario garantizar el cumplimiento de este derecho que también afecta a los demás ciudadanos, de manera indirecta porque que al acudir con sus familiares no tienen el trato que esperaban para su atención, pese en muchos casos la gravedad de su estado de salud.

Dentro de la investigación realizada los resultados obtenidos a través de las encuestas denotan la falta de seguridad y credibilidad que tienen al acudir a este centro de salud, ya que esperan como no ser atendidos, y muchas veces pese a sus aportaciones otorgadas en dicha institución prefieren acudir a centros privados o particulares para una mejor atención.

Si consideramos la sección segunda del Art. 359 de la Constitución de la República del Ecuador que textualmente dice: El sistema nacional de salud comprenderá las instituciones, programas, políticas, recursos, acciones y actores en salud; abarcará todas las dimensiones del derecho a la salud; garantizará la promoción, prevención, recuperación y rehabilitación en todos los niveles; y propiciará la participación ciudadana y el control social. (Constitución, 2018)

Esto nos permite analizar que el Estado está comprometido en presentar planes que beneficien la salud de los ecuatorianos a través de sus políticas públicas, pero que las autoridades que están frente a los mismo no le dan la debida atención, es por esta razón que dentro del hospital del Instituto Ecuatoriano de Seguridad Social de la ciudad de Santo Domingo, por no acotar con la disposición dadas para satisfacer las necesidades del grupo prioritario (adultos mayores) en lo que respecta a su atención es que se encuentra con este caos de vulneración de un derecho muy importante como es el de la salud. 


\section{CONCLUSIONES}

El Estado como garantista de los derechos de los ciudadanos y en especial del grupo de atención prioritaria (adultos mayores), debe de exigir el cumplimiento de la norma Constitucional por parte de las autoridades correspondientes que se encuentran frente de la administración de los centros de salud, para evitar vulneración del derecho fundamental e importante como el de la salud.

Se debe considerar la propuesta dentro del artículo científico, lo que permitirá un resultado para descongestionar los hospitales del Instituto de Seguridad Social en especial el del cantón Santo Domingo, y con el que se permitirá una mayor afluencia de usuario de adultos mayores en sus horarios establecidos.

Así mismo los adultos mayores reciban la atención adecuado con profesionales de la salud especializados en materia de geriatría dentro del lugar de su residencia, sin que tenga la desventaja de trasladarse a otra ciudad para recibir su tratamiento o atención médica, ya que por su dificultad de movilización resulta afectada más su salud. Que debemos considerar que todos vamos a la etapa de la vejez y que debemos considerar que se deben de respetar sus derechos que se encuentran en la Constitución de la Republica, por lo que no se debe de permitir su vulneración, sino más bien buscar lineamientos que permitan un mejoramiento y cumplimiento de los mismos. En conclusión, se establece la necesidad de entablar los canales de atención prioritaria y canal de capital humano, cuyo resultado es la existencia de personal capacitado en atención de enfermedades degenerativas y catastróficas en el ámbito geriátrico

\section{FINANCIAMIENTO}

No monetario.

\section{AGRADECIMIENTO}

A la Universidad Regional Autónoma de los Andes; por motivar el desarrollo de la Investigación. 


\section{REFERENCIAS CONSULTADAS}

Asamblea Nacional Constituyente de la República del Ecuador, (2008). Constitución de la República del Ecuador. Montecristi. Registro Oficial 449 de 20-oct-2008. Recuperado de https://n9.cl/sia

Batista-Pereda, Y., Yera-Alós, I., Martínez-Martín, M., Pérez-Jiménez, D., \& ArandaCanosa, S. (2016). El análisis de la situación de salud hospitalaria: herramienta para la gestión de hospitales del Instituto Ecuatoriano de Seguridad Social. [The analysis of the hospital health situation: tool for the management of hospitals of the Ecuadorian Institute of Social Security] Revista Médica Electrónica, 38(4), 530-542. Recuperado en 21 de enero de 2021, de https://n9.cl/ny96j

Bustamante, M., Lapo, María del C., Torres, J., \& Camino, S. (2017). Factores Socioeconómicos de la Calidad de Vida de los Adultos Mayores en la Provincia de Guayas, Ecuador. [Socioeconomic Factors in the Quality of Life of Older Adults in Guayas Province, Ecuador] Información Tecnológica, 28(5), 165176. https://dx.doi.org/10.4067/S0718-07642017000500017

Congreso Nacional (1992) Ley de Anciano. [Elder Law]. Decreto Ejecutivo 3437 Registro Oficial 961 de 19-6-1992

Congreso Nacional del Ecuador (2001) Ley de Seguridad Social. [Social Security Act]. Registro Oficial Suplemento 465 de 30-nov-2001. Recuperado de: https://n9.cl/af5t5

Chóez-Chiliquinga, E., Zambrano-Pérez, M., Barros-Carvajal, M., \& Parra-Parra, M. (2019). La violencia hacia el adulto mayor y sus consecuencias. [Violence against the eldest adult and its consequences]. Ciencia Digital, 3(1.1), 99-107. https://doi.org/10.33262/cienciadigital.v3i1.1.362

Ministerio de Inclusión Económica y Social (2013). Informe de gestión 2012-2013. [Management Report 2012-2013]. Recuperado de: https://www.inclusion.gob.ec/publicaciones

Organización de Estados Americanos (2012). Convención interamericana sobre la protección de los derechos humanos de las personas mayores. [Inter-American Convention on the Protection of the Human Rights of The Elderly]. Recuperado de: https://n9.cl//zodf

Organización Mundial de la Salud (2002). Envejecimiento activo: un marco político. [Active ageing: a political framework]. Recuperado de: https://n9.cl/o6i6r 
lustitia Socialis. Revista Arbitrada de Ciencias Jurídicas.

Año V. Vol. V. №3. Edición Especial. 2020-III:

Universidad Regional Autónoma de los Andes

Hecho el depósito de Ley: FA2016000064

ISSN: 2542-3371

FUNDACIÓN KOINONIA (F.K). Santa Ana de Coro, Venezuela

Marco Fernando Saltos-Salgado; Carlos Alfonso Grijalva-Terán

Rodríguez-Quintana, T., Ayala-Chauvin, M., Ortiz-Rosero, M., Ordoñes-Paccha, M., Fabelo-Roché, J. \& Iglesias-Moré, S. (2019). Caracterización de las condiciones de salud de los adultos mayores en Centros Geriátricos de la ciudad de Loja. Ecuador, 2017. [Characterization of the health conditions of older adults in Geriatric Centers of the city of Loja. Ecuador, 2017] Revista Habanera de Ciencias Médicas, 18(1), 138-149. Recuperado de: https://n9.cl/iieg

C2020 por los autores. Este artículo es de acceso abierto y distribuido según los términos y condiciones de la licencia Creative Commons Atribución-NoComercial-Compartirlgual 4.0 Internacional (CC BY-NC-SA 4.0)

(https://creativecommons.org/licenses/by-nc-sa/4.0/). 